\title{
Novo gênero e espécie de Portanini Linnavuori, e notas taxonômicas (Hemiptera, Cicadellidae, Xestocephalinae)
}

\author{
Adenomar Neves de Carvalho ${ }^{1} \&$ Rodney Ramiro Cavichioli ${ }^{2}$
}

${ }^{1}$ Faculdade Adventista Paranaense, C. Postal 1528, 87001-970, Maringá-PR. adenomarc@yahoo.com.br

${ }^{2}$ Departamento de Zoologia, Universidade Federal do Paraná, Caixa Postal 19020, 81531-990 Curitiba-PR. Bolsista CNPq, cavich@ufpr.br

\begin{abstract}
New genus and species of Portanini Linnavuori, and taxonomic notes (Hemiptera, Cicadellidae, Xestocephalinae). A new genus and a new species of Portanini are described: Paraportanus gen. nov. and the type species Paraportanus jenniferae sp. nov. (from Brazil, Maranhão). Nine species formerly described in Portanus Ball, 1932 are transferred into this new genus: Paraportanus longicornis (Osborn, 1923) comb. nov. = Portanus chelatus DeLong, 1976 syn. nov.; Paraportanus elegans (Kramer, 1961) comb. nov.; Paraportanus facetus (Kramer, 1961) comb. nov.; Paraportanus eburatus (Kamer, 1964) comb. nov.; Paraportanus filamentus (DeLong, 1980) comb. nov.; Paraportanus bicornis (Carvalho \& Cavichioli, 2003) comb. nov.; Paraportanus bimaculatus (Carvalho \& Cavichioli, 2003) comb. nov.; Paraportanus cinctus (Carvalho \& Cavichioli, 2003) comb. nov.; Paraportanus variatus (Carvalho \& Cavichioli, 2003) comb. nov. New geographical distribution data are recorded for P. facetus; P. elegans e P. longicornis. A key is presented.
\end{abstract}

KEYWORDS. Leafhoppers; Paraportanus; description; Taxonomy.

RESUMO. Novo gênero e espécie de Portanini Linnavuori, e notas taxonômicas (Hemiptera, Cicadellidae, Xestocephalinae) Nove espécies são transferidas de Portanus Ball, 1932 para Paraportanus gen. nov.: Paraportanus longicornis (Osborn, 1923) comb. nov. = Portanus chelatus DeLong, 1976 syn. nov.; Paraportanus elegans (Kramer, 1961) comb. nov.; Paraportanus facetus (Kramer, 1961) comb. nov.; Paraportanus eburatus (Kamer, 1964) comb. nov.; Paraportanus filamentus (DeLong, 1980) comb. nov.; Paraportanus bicornis (Carvalho \& Cavichioli, 2003) comb. nov.; Paraportanus bimaculatus (Carvalho \& Cavichioli, 2003) comb. nov.; Paraportanus cinctus (Carvalho \& Cavichioli, 2003) comb. nov.; Paraportanus variatus (Carvalho \& Cavichioli, 2003) comb. nov.. Novos dados de distribuição geográfica são registrados para: $P$. facetus; $P$. elegans e $P$. longicornis. Chave para identificação das espécies é apresentada.

PALAVRAS-CHAVE. Cigarrinhas; Paraportanus; descrição; Taxonomia.

Xestocephalinae constitui um grupo pequeno, incluindo seis gêneros: Xestocephalus Van Duzee, 1892; Myrmecophryne Kirkald, 1906c; Matsumurana Distant, 1917, Ootacamundus Distant, 1918; Portanus Ball, 1932 e Aloxestocephalus Evans, 1973 (Oman et al., 1990; Nielson \& Knight, 2000). Os membros desse grupo estão entre os menores cicadelídeos, com tamanho dos adultos variando entre 4,0 e 7,0mm. Linnavuori (1959), ao revisar a subfamília, propôs sua divisão em duas tribos: Xestocephalini, com representantes distribuídos em todas as regiões zoogeográficas (com exceção da Europa e da maior parte da Ásia), e Portanini, na região Neotropical.

Portanini contém um gênero (Portanus Ball, 1932) e 52 espécies. As espécies dessa tribo podem ser diferenciadas das de Xestocephalini, pelas seguintes características, segundo Linnavuori (1959): (1) corpo longo e delgado; (2) face alongada; (3) fronte retangular; (4) clípeo com lados proximamente paralelos; (5) loros grandes, estendendo-se até o ápice do clípeo; (6) antenas usualmente longas, quase tão longas quanto o corpo; (7) lóbulos supra-antenais, em vista lateral, carenados e oblíquos; (8) coroa triangularmente projetada; (9) sutura coronal longa e (10) estilos com ápice alargado e bífido.

No presente trabalho um novo gênero e uma nova espécie são propostos; uma sinonímia e novas combinações são apresentadas.

As siglas citadas no texto correspondem: INPA - Instituto Nacional de Pesquisas da Amazônia; DZUP - Departamento de Zoologia, UFPR, Coleção de Entomologia Pe. Jesus Santiago Moure; OSUC - Ohio State University Collection; MPEG - Museu Paraense Emílio Goeldi e CMNH - Carnegie Museum of National History.

\section{Paraportanus gen. nov.}

\section{Espécie-tipo: Paraportanus jenniferae sp. nov.}

Comprimento: 5,3-7,0mm.

Diagnose. Pigóforo do macho fortemente pronunciado portando, na maioria dos casos, um par de processos espiniformes na margem póstero-ventral. Placas subgenitais triangulares com estreitamento apical, sem linha despigmentada transversa no terço basal. Conetivo em forma de "V".

Cabeça, em vista dorsal, com coroa (Fig. 1) pronunciada anteriormente, com margem anterior arredondada, sem carena na transição com a face; comprimento mediano variando entre $1 / 2$ e 1/3 da largura da cabeça; superfície microesculturada; 
ocelos na margem anterior da coroa, eqüidistantes do ângulo anterior dos olhos e da linha mediana da coroa; sutura coronal longa, atingindo a metade do comprimento da coroa; lóbulos supra-antenais, em vista lateral, com as margens oblíquas e carenadas. Fronte mais longa que sua maior largura, em vista lateral, fracamente intumescida; suturas frontogenais com reentrância na altura das antenas, convergindo para o ápice, tocando os ocelos; sutura epistomal completa; loros grandes, atingindo a margem apical do clípeo; clípeo, em vista frontal, com as margens paralelas ou divergentes em relação à base; antenas longas, ultrapassando a metade do comprimento das asas anteriores, atingindo ou não o seu ápice.

Tórax. Pronoto (Figs. 1 e 7), em vista dorsal, com largura variável (igual ou maior do que a largura da cabeça); margens laterais arredondadas ou estreitamente arredondadas; margem posterior retilínea; disco do pronoto com puncturas contornadas por áreas claras; carena notopleural completa. Escutelo mais largo basalmente do que longo. Asas anteriores quatro vezes mais longas do que sua maior largura, com três células anteapicais fechadas; célula anteapical mediana maior que as adjacentes; terceira e quarta células apicais subretangulares; sem veias extranumerárias e plexo de veias; apêndice reduzido. Pernas posteriores com fórmula femural 2.2.1.

Genitália do macho. Pigóforo (Fig. 2), em vista lateral, pronunciado com a margem posterior variável; macrocerdas curtas e delgadas distribuídas na porção médio-dorsal; com ou sem processos; margem ventral com forte reentrância no terço basal. Placas subgenitais (Fig. 3), em vista ventral, triangulares com distinto estreitamento apical, com uma fileira de macrocerdas oblíqua à margem interna. Estilos alongados (Figs. 4 e 8), em vista dorsal, delgados, com ápice afilado e levemente retorcido ou alargado e bífido. Conetivo curto, (Figs. 4 e 8), vista dorsal, robusto e em "V". Edeago (Figs. 5 e 9), em vista lateral, muito variável, geralmente delgado com a porção apical fortemente curvada para cima; com processos apicais ou pré-apicais; com ou sem apódema no terço basal. Gonóporo apical.

Coloração. A coloração do dorso é variável entre as espécies, não apresentando um padrão para o gênero.

Fêmea. Comprimento total 5,6-7,0mm. Morfologia externa semelhante à do macho.

Genitália. Esterno VII (Fig. 10) geralmente tão largo quanto longo; com margem posterior de forma variável; margens laterais paralelas. Esterno VIII completamente membranoso. Pigóforo (Fig. 11), em vista lateral, moderadamente pronunciado posteriormente; ápice agudo; macrocerdas distribuídas na porção apical, geralmente estendendo-se anteriormente ao longo da margem ventral. Primeira valva do ovipositor (Fig. 12), em vista lateral, curvada dorsalmente com metade apical levemente alargada e ápice agudo; área esculturada dorsal estendendo-se por quase toda a área expandida; diminutas cerdas presentes ao longo do seu comprimento, sendo mais numerosas na porção basal. Segunda valva do ovipositor (Fig. 13), em vista lateral, curvada dorsalmente com metade apical moderadamente alargada, estreitando-se gradualmente em direção ao ápice, este agudo; margem dorsal com cerca de 30 dentes arredondados e contíguos, distribuídos do início da porção expandida à porção apical; dentes com ou sem dentículos na margem posterior. Terceira valva do ovipositor (Fig. 14), em vista lateral, longa, com ápice agudo; metade basal distintamente mais estreita que a apical; porção apical com um pequeno número de cerdas e diminutas estruturas espiniformes que se estendem para a base ao longo da margem ventral.

Discussão. Uma análise filogenética morfológica do gênero Portanus foi apresentada por Carvalho \& Cavichioli (2006). Desta análise resultou uma única árvore mais parcimoniosa (123 passos, IC $=0,75$ e IR $=0,88$ ) que embora sustentasse a monofilia do gênero, apresentou evidências de dois clados bem definidos: o primeiro, mais basal, composto por $\left(_{(()(((P \text {. }}\right.$ eburatus $+P$. longicornis) $P$. sp. nov. 1) P. elegans) $P$. facetus $)$ $P$. bicornis $) P$. variatus $)+((P$. cinctus $+P$. bimaculatus $) P$. filamentus)) sendo sustentado por três sinapomorfias: (1) margem ventral do pigóforo com forte reentrância no terço basal; (2) placa subgenital triangular com a porção apical estreitada e (3) conetivo em forma de um "V" invertido (Bootstrap $=85$ e Suporte de Bremer $=4)$. O segundo, mais apical, composto por P. stigmosus (espécie-tipo do gênero) e demais espécies de Portanus incluídas, formando um clado sustentado por três sinapomorfias, sendo duas delas exclusivas: (1) placa subgenital com uma linha despigmentada transversa no terço basal; (2) conetivo com um processo basiventral curto e (3) estilo com a porção apical bífida e o ramo interno perpendicular a paralelo ao externo. Esse caráter revertendo para o estado (convergente) em $P$. cephalatus, $P$. caudatus, $P$. retusus, $P$. avis, $P$. youngi, $P$. minor, $P$. major, $P$. lineatus, $P$. spiniloba, $P$. acerus, $P$. quadrinus, $P$. dentatus, $P$. telmae, P. pictus, P. uhleri, P. boliviensis, P. digitus, P. eliasi, P. macullatus, $P$. hasemani, P. lex, $P$. linnavuorii e P. stigmosus (Bootstrap $=70$ Suporte de Bremer $=2)$.

As espécies de Paraportanus gen. nov. apresentam uma grande diversidade no padrão de coloração do corpo que dificulta o seu reconhecimento quando se observa apenas este caráter. Por outro lado, a genitália do macho mostra um padrão constante na morfologia fornecendo características diagnósticas para as espécies. Entretanto, a genitália da fêmea não possui tal padrão. A forma do sétimo esterno e da segunda valva do ovipositor variam bastante entre as espécies impossibilitando estabelecer um padrão que possa identificar as fêmeas.

O gênero Paraportanus pode ser dividido em dois grupos: 1) espécies com a coroa pouco pronunciada anteriormente, olhos aproximadamente globulares (Fig. 7), estilo com a porção apical afilada e retorcida (Fig. 8), edeago parcialmente fusionado ao conetivo (Fig. 9) e, 2) espécies com a coroa mais alongada, com olhos aproximadamente elípticos (Fig. 1), estilo com a porção apical alargada e bífida (Fig. 4) e edeago completamente articulado ao conetivo (Fig. 5).

A presença de um par de processos ventrais na porção póstero-ventral do pigóforo do macho é uma característica 
marcante para muitas espécies (P. filamentus, P. eburatus, $P$. longicornis, $P$. elegans e $P$. jenniferae $\mathbf{s p . ~ n o v . . ~ T a i s ~ p r o c e s s o s ~}$ em $P$. variatus, quando observados em vista ventral, têm forma de pequenos dentes arredondados e voltados medianamente. Outra característica diagnóstica é a presença de um apódema simples na haste do edeago, com exceção de $P$. filamentus (Fig. 9), que pode variar na forma e posição.

Chave para as espécies de Paraportanus gen. nov.

1. Estilo com porção apical afilada e levemente retorcida ..... 2 Estilo com porção apical alargada e bífida ... ... 4

2. Pigóforo com margem posterior truncada com processo espiniforme longo, robusto no ângulo póstero-ventral, curvado dorso-lateralmente .......... P. filamentus (Brasil).

Pigóforo com margem posterior angulada sem processo no ângulo póstero-ventral

3. Coroa alaranjada com duas manchas pretas subtriangulares entre os ocelos . P. cinctus (Brasil).

Coroa marrom com manchas alaranjadas P. bimaculatus (Brasil).

4. Edeago, em vista lateral, com um par de processos lamelares agudos, voltados posteriormente

Edeago, em vista lateral, com um par de processos espiniformes agudos, voltados ventralmente ou posteriormente .. .7

5. Conetivo com processo lamelar na confluência ventral dos braços; pigóforo com processo espiniforme extremamente longo, delgado e curvado dorsalmente, ultrapassando o ângulo póstero-dorsal .......................... 6

Conetivo não como acima; pigóforo com processo espiniforme curto, robusto e curvado dorsalmente, porém não ultrapassando o ângulo póstero-dorsal ........ P. eburatus (Brasil e Panamá).

6. Coroa e pronoto marrom-claros, minuciosamente marcados com manchas amarelo-palha circulares ou alongadas ..... . P. longicornis (Bolívia e Brasil).

Coroa alaranjada com um par de faixas largas horizontais marrom-escuras, atrás dos ocelos; pronoto com manchas amarelo-palha circulares ou alongadas, porém com um distinto par de manchas alaranjadas na porção central do disco P. elegans (Brasil e Venezuela).

7. Pigóforo com processo espiniforme alongado no ângulo póstero-ventral, curvado dorsalmente; edeago com processo pré-apical curto, voltado ventralmente

P. jenniferae sp. nov. (Brasil).

Pigóforo sem processo espiniforme no ângulo pósteroventral; edeago com processo pré-apical curto ou alongado, voltado para trás
8. Edeago com um par de processos espiniformes extremamente longos ....... P. facetus (Brasil e Venezuela).

Edeago com um par de processos espiniformes curtos ..... 9

9. Pigóforo, em vista lateral, com a porção apical angulada. Em vista ventral, com processo dentiforme curto e robusto na porção pré-apical, voltado medianamente. Escutelo marrom com a porção apical branca

P. variatus (Brasil e Peru).

Pigóforo, em vista lateral, com a porção apical truncada. Sem processo na porção pré-apical. Escutelo marrom com duas manchas brancas na margem lateral

P. bicornis (Brasil)

\section{Paraportanus jenniferae sp. nov.}

Figs. 1-6

Holótipo macho. Comprimento total 5,7mm. Cabeça (Fig. 1), em vista dorsal, com margem anterior arredondada; coroa de comprimento mediano aproximadamente $1 / 3$ da largura da cabeça. Clípeo retangular com ápice alargado; margem apical retilínea. Pronoto mais largo que a cabeça. Asas anteriores com venação evidente; célula anteapical mediana maior que as adjacentes; terceira célula apical retangular e a quarta subretangular. Pigóforo (Fig. 2), em vista lateral, com margem posterior angulada; com processo espiniforme longo, robusto e fortemente esclerotizado na margem póstero-ventral, este está curvado dorso-lateralmente. Placas subgenitais (Fig. 3), em vista ventral, triangulares com a porção apical levemente estreitada em direção ao ápice. Conetivo (Fig. 4), em vista dorsal, em forma de "V", com processo lamelar dorsal, próximo do ponto de articulação com o edeago. Estilos, (Fig. 4) em vista dorsal, com ápice alargado e bífido; ramo interno agudo e perpendicular ao externo. Edeago (Fig. 5), em vista lateral, com curvatura dorsal; com dois processos espiniformes préapicais; estes (Fig. 6), em vista ventral, subcilíndricos e alongados; com apódema simples no terço basal. Gonóporo apical.

Coloração. Face, coroa e pronoto, marrom-escuros, manchados com diversas áreas ovais ou circulares amarelopalha, conferindo um aspecto reticulado. Escutelo branco com ângulos marrom-escuros. Asas anteriores marrom-claras, subhialinas, com veias densamente manchadas de branco; grande área translúcida em forma de zig-zag na base das células préapicais; porção apical mais escura com grandes áreas translúcidas na base das células apicais.

Fêmea. Comprimento total 7,0mm. Morfologia externa semelhante à do macho.

Esterno VII retangular com a margem posterior sinuada e com distinto dente mediano. Segunda valva do ovipositor, em vista lateral, moderadamente expandida na metade apical; com haste estreitando-se em direção ao ápice, este agudo; com 28 dentes conspícuos distribuídos na margem dorsal da porção expandida da valva, sem dentículos.

Nota taxonômica. Paraportanus jenniferae sp. nov. (tratada como $P[$ ortanus $]$. sp. nov. 1 no estudo filogenético apresentado 

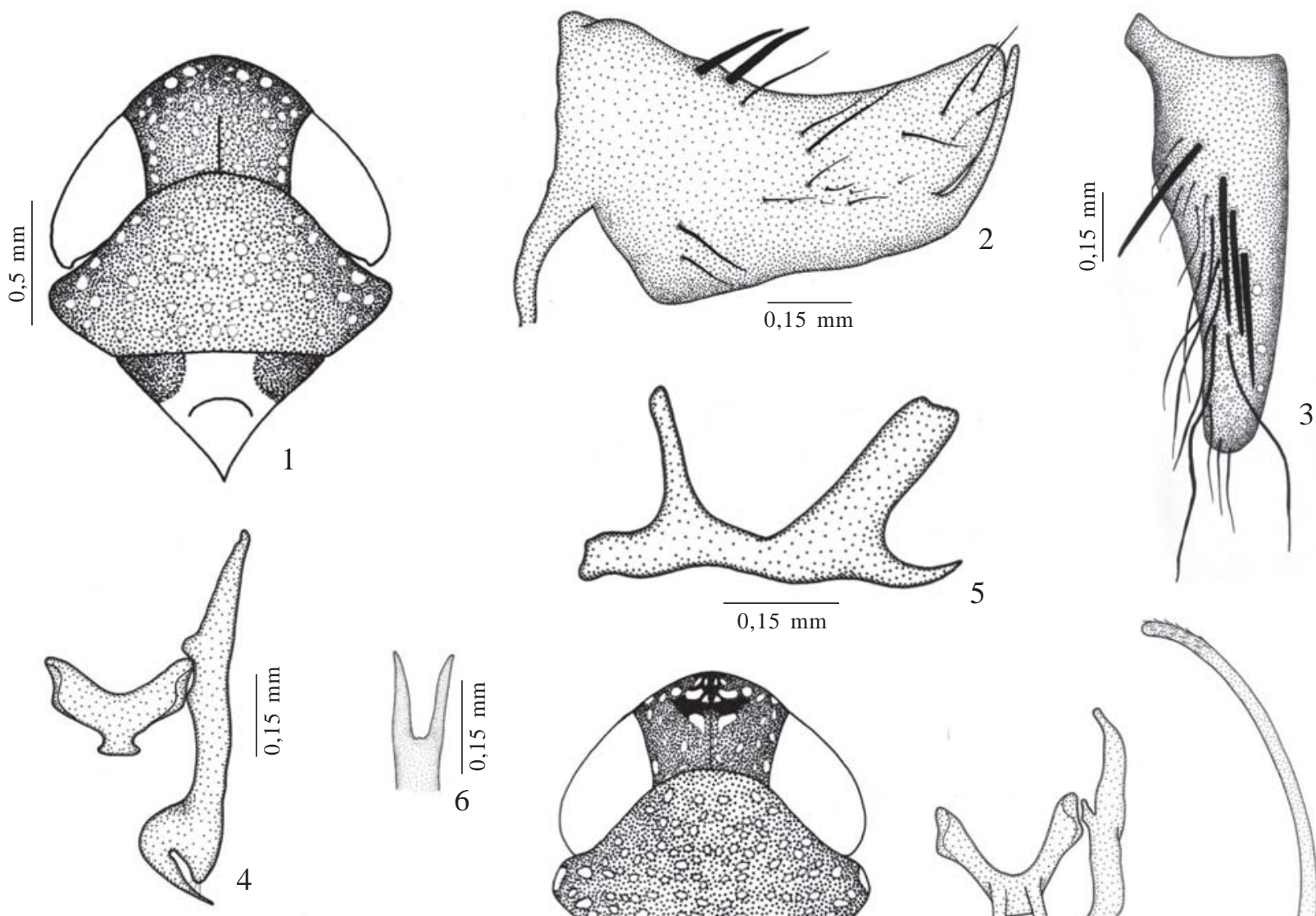

3
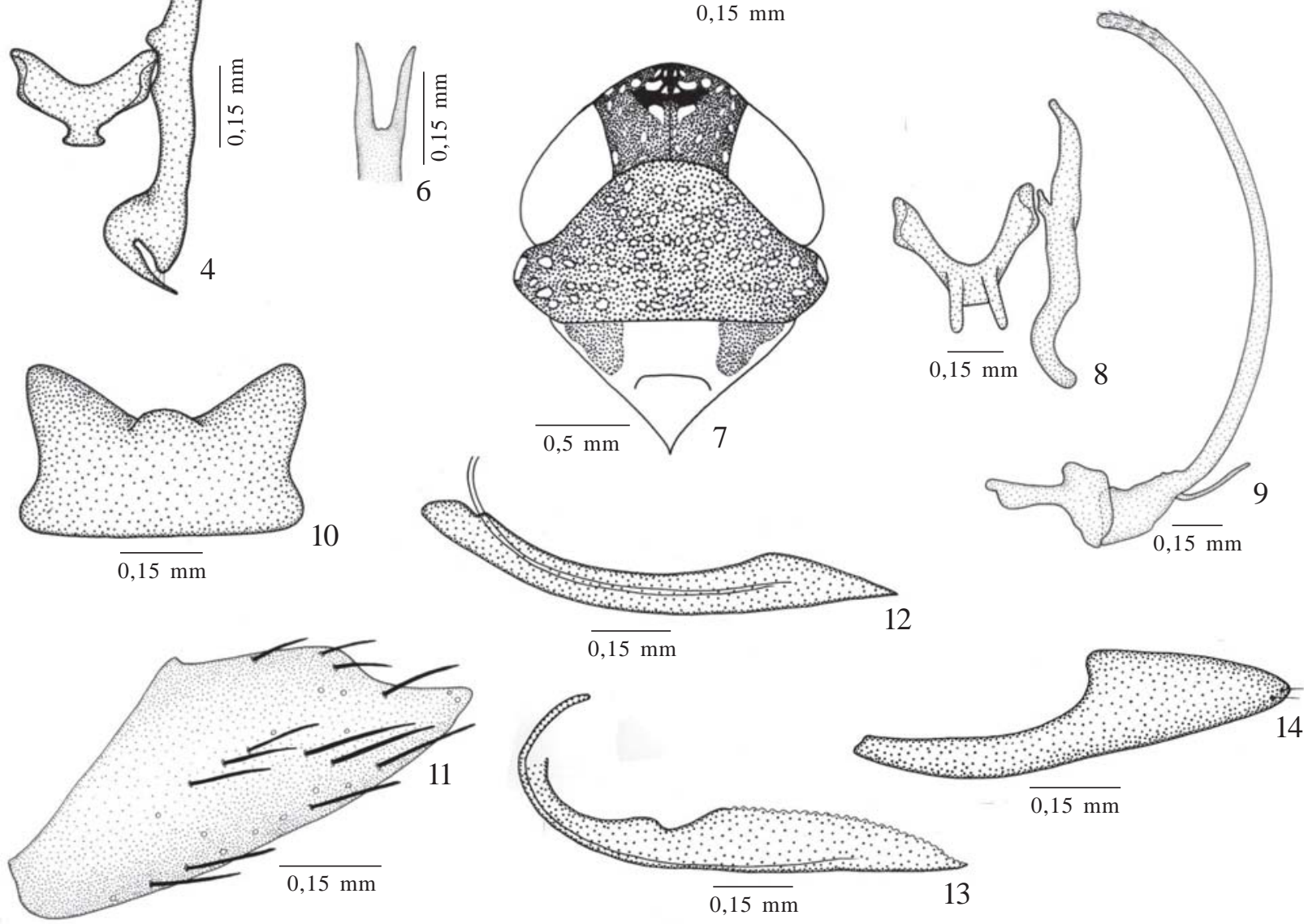

Figs. 1-14. 1-6, Paraportanus jenniferae: 1, cabeça, pronoto e escutelo, vista dorsal; 2, pigóforo, vista lateral; 3, placa subgenital, vista ventral; 4, estilo e conetivo, vista dorsal; 5, edeago, vista lateral; 6, porção apical do edeago, vista ventral. 7-9, Paraportanus filamentus: 7, cabeça, pronoto e escutelo, vista dorsal; 8, estilo e conetivo, vista dorsal; 9, edeago, vista lateral. 10-14, Paraportanus elegans: 10, Sétimo Esterno, vista ventral; 11, pigóforo feminino, vista lateral; 12, primeira valva do ovipositor, vista lateral; 13, segunda valva do ovipositor, vista lateral; 14, terceira valva do ovipositor, vista lateral.

por Carvalho \& Cavichioli (2006)), pode ser distinguida das outras espécies conhecidas do gênero pela seguinte combinação de características: 1) pigóforo do macho (Fig. 2) com margem posterior angulada e curvada para cima; 2) ângulo póstero-ventral do pigóforo com um processo agudo alongado e curvado dorsalmente, ultrapassando ou não o ápice; 3 ) haste do edeago (Fig. 6) com apódema basal longo e 4) com porção apical fortemente curvada para cima com um par de processos 
espiniformes curtos, agudos e voltados para trás, no terço apical, podendo ser retilíneos ou com ápices curvados lateralmente.

O pigóforo do macho é muito similar aos de $P$. longicornis e P. eburatus. Tem, em ambas as espécies, um processo alongado no ângulo póstero-ventral, fortemente curvado póstero-dorsalmente. A forma do edeago de $P$. jenniferae $\mathbf{s p}$. nov. (Fig. 5) também é similar de P. longicornis e P. eburatus. O edeago, nessas espécies, é curvado póstero-dorsalmente e o apódema basal bem desenvolvido. $P$. jenniferae sp. nov., $P$. longicornis e $P$. eburatus possuem um par de processos agudos laterais na porção pré-apical da haste do edeago. Esses processos são curvados póstero-dorsalmente, em vista ventral, distintamente espiniformes na primeira espécie, enquanto nas demais, apresentam-se lamelares e distalmente alargados e truncados.

O esterno abdominal VII da fêmea de P. jenniferae sp. nov. é similar ao de P. eburatus com a margem apical projetada com um distinto dente mediano. Entretanto, em P. jenniferae sp. nov. a margem é acentuadamente projetada posteriormente.

Holótipo ơ BRASIL. Maranhão: Imperatriz / Ribeirãozinho / 0104.VIII.1989 // Brasil MA / F. F. Ramos, leg. Armadilha / Malaise // Holótipo. Parátipos: $20^{x}$, com os mesmos dados do holótipo. $30^{x} \mathrm{e} 4$ 우, Brasil Acre / Porto Acre, Humalta / 15.VI-02.VII.1992 // Garayeb, Pena, Henrique \& Edimar leg. // Armadilha / Malaise // Homoptera / Auchenorrhyncha / Cicadeloidea: Cicadellidae. Incorporação: 30.IV.1999. 10َ', Brasil Rondônia / Ouro Preto d'Oeste / 11-13.XI.1984 / Armadilha / Malaise (DZUP). 10, Ibdem, 07-10.IV.1985 // Armadilha / 1,6 m suspensa / Malaise // Brasil Rondônia / W. França, leg. 1 $0^{\star}$, Brasil Acre / Porto Acre, Humalta / 15.VI-02.VII.1992 // Garayeb, Pena, Henrique \& Edimar leg. // Armadilha / Malaise // Homoptera / Auchenorrhyncha / Cicadeloidea: Cicadellidae / Incorporação: 30.IV.1999. (MPEG).

Etimologia. A espécie é dedicada à Jennifer S. de Carvalho, filha do primeiro autor.

\section{Alterações taxonômicas}

Relacionamos abaixo as espécies transferidas no presente estudo para Paraportanus, a partir da interpretação da árvore filogenética apresentada por Carvalho \& Cavichioli (2006).

\section{Paraportanus longicornis (Osborn) comb. nov.}

Scaphoideus longicornis Osborn, 1923:37-38.

Portanus longicornis; Ball, 1932:18.

Portanus chelatus DeLong, 1976:38 Syn. nov.

Material-examinado. Holótipo macho de Scaphoideus longicornis Osborn, 1923 (CMNH): Holótipo de Portanus chelatus DeLong, 1976. BRASIL. Roraima - Ilha de Maracá (Rio Uraricoera), $2 \sigma^{*}, 21$ -

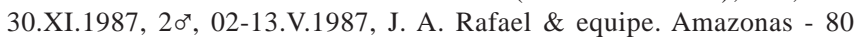
Km de Manaus, 1 (sem abdome), 13.XI.1985, Bert Klein Col.. Pará Campo da Palha, 10, 06.XII.1988, I. S. Gorayeb, Col. (INPA). Mato Grosso - Cáceres, $10^{\star}$, 30.XII.1984, C. Elias Leg.; Chapada dos Guimarães, 10 $0^{\star}$, 13-17.II.1986, I, S. Gorayeb, Col. (INPA); Nova Xavantina, $1 \sigma^{x}$, 31.XII.98, 10*, 25.V.98, $1 \sigma^{x}$, 27.II.98, Cabette, H. S. R. Col., 1ㅇ, 07.VII.97, Barreira, R. L. Col.. Minas Gerais - Belo Horizonte, 10, 29.X-04-XI.91, A. F. Kumagai, Col.. Ceará - Barbalha, 10, V.1969, M. Alvarenga, Col. (DZUP). Rondônia - Ouro Preto do Oeste, 10, 1113.VI.1984, F. F. Ramos, Col. (INPA) (registro novo).
Paraportanus elegans (Kramer) comb. nov.

Portanus elegans Kramer, 1961:236.

Material-examido. Holótipo macho (USNM). BRASIL / Rondônia - Vilhena /20 $0^{x}, 13.12 .1986 / / 10^{x}$ e 1우 17.X.1986 // 1ㅇ, 10.XI.1986 // C. Elias leg / Polonoroeste // Carvalho, A. N. Det. // (DZUP); Ariquemes / 30, 28.X.1986 / J. A. Rafael leg. // Carvalho, A. N. Det. // (INPA). Amazonas - Humaltá / (sem abdome) / 54 Bis, 17-21.IX.1990 / Malaise $\left(60^{\pi}\right) /$ R. Constantino leg. / / (DZUP); Pamajaú / $10^{x}$ e 1우, (sem data de coleta) / P. N. Jaú / Campina arbustiva // Carvalho, A. N. Det. // (INPA) Mato Grosso - Sinop / 10 $\sigma^{\star}$ X.1975 / M. Alvarenga leg. // Carvalho, A N. Det. // (DZUP). Pará - Óbidos / $10^{\star}$ e 1ㅇ, 29.VIII.2001 / Sítio Curió, 014703S 550705W / Malaise / J. A. Rafael \& J. Vidal leg.// Carvalho, A. N. Det. // (INPA) (registro novo).

\section{Paraportanus facetus (Kramer) comb. nov.}

Portanus facetus Kramer, 1961:236.

Material-examinado. Holótipo macho (USNM). BRASIL / Rondônia - Ouro Preto d'Oeste / 10, 03.IX.1987 / C. Elias, leg.// Carvalho, A. N. Det. // (DZUP). Amazonas - Manaus $\left(2^{\circ} 25^{\prime} \mathrm{S}, 60^{\circ} \mathrm{O}\right) / 10^{7}, 27 . \mathrm{XI} .1985$ / Bert Klein Col./ Malaise // Carvalho, A. N. Det. // (INPA) (registro novo).

Paraportanus eburatus (Kramer) comb. nov.

Portanus eburatus Kramer, 1964:7.

Material-examinado. Holótipo macho (USNM). BRASIL. Serra do Navio / II.7.1961 // Brasil / J. \& B. Bechyne // + // Portanus / eburatus (DZUP) (registro novo).

\section{Paraportanus filamentus (DeLong) comb. nov. \\ Portanus filamentus DeLong, 1980:82.}

Material-examinado. Holótipo macho (OSUC).

Paraportanus bicornis (Carvalho \& Cavichioli) comb. nov. Portanus bicornis Carvalho \& Cavichioli, 2003:558.

Material-examinado. Holótipo macho (DZUP).

Paraportanus bimaculatus (Carvalho \& Cavichioli) comb. nov.

Portanus bimaculatus Carvalho \& Cavichioli, 2003:553.

Material-examinado. Holótipo macho (DZUP).

Paraportanus cinctus (Carvalho \& Cavichioli) comb. nov. Portanus cinctus Carvalho \& Cavichioli, 2003:550.

Material-examinado. Holótipo macho (DZUP).

Paraportanus variatus (Carvalho \& Cavichioli) comb. nov. Portanus variatus Carvalho \& Cavichioli, 2003:548.

Material-examinado. Holótipo macho (DZUP). BRASIL. Sinop Mato Grosso / Br X.1975 / M. Alvarenga, leg. (DZUP). BRASIL / Amazonas: São Gabriel da Cachoeira / (01 $05^{\circ}$ N-69 $\left.51^{\circ} \mathrm{W}\right), 2 \sigma^{\top}$, 05.IV21.VI.1993 / Motta, C. S., Ferreira, R. L. Vidal, J. \& Matteo, B., leg. / / (INPA). Acre: Porto Acre (Humalta) / $2 \sigma^{x}$ e 1우 15.VI-02.VII.1992 / Armadilha suspensa $(20 \mathrm{~m}) /$ Garayeb, Pena Henriques \& Edmar, leg. Pará - Serra Norte (Estrada da serraria) / 10 $0^{x}, 10-15$.IX.1983 / F. F. 
Ramos, leg.. Rondônia: Ji-Paraná / 10 , 17-20.XI.1984 / Armadilha Malaise; Ibidem,1ㅇ, 20-23.XI.1984. Ouro Preto d'Oeste (Linha 62 Km 16) / 20', 13-15.XI.1984 / Armadilha suspensa, 1,6 m // (MPEG). PERU: M. de Dio, Parque Manu / Pektiza 340 m / (11 ${ }^{\circ} 55^{\prime} 48$ "S$\left.71^{\circ} 15^{\prime} 18^{\prime \prime} \mathrm{W}\right) / 10^{\star}, 16 . X .1991 / \mathrm{M}$. Casagrande, leg.// (DZUP) (registro novo).

Agradecimentos. Aos curadores Stuart H. McKamey (USNM), Chen Young (CMNH), A. Henriques (INPA) e Norman Johnson e Luciana Musetti (OSUC) pelo empréstimo do material-tipo. À Coordenação de Aperfeiçoamento de Pessoal Em nível Superior - CAPES, pela concessão da bolsa de doutorado e ao Conselho Nacional de Desenvolvimento Científico e Tecnológico - CNPq, pela bolsa de produtividade em pesquisa concedida ao segundo autor.

\section{REFERÊNCIAS}

Ball, E. D. 1932. New genera and species of leafhoppers related to Scaphoideus. Washington Academy of Science 23: 531-532.

Blocker, H. D. \& B. W. Triplehorn. 1985. External morphology of leafhoppers. In: L. R. Nault \& J. G. Rodrigues (eds.). The leafhoppers and Planthoppers. John Wiley \& Sons, New York, 500 p. (p. 41-60).

Carvalho, A. N. \& R. R. Cavichioli. 2001. Portanus Ball: descrições de cinco espécies novas (Hemiptera, Auchenorryncha, Cicadellidae, Xestocephalinae) do Mato Grosso, Brasil. Revista Brasileira de Zoologia 18: 855-867.

Carvalho, A. N. \& R. R. Cavichioli. 2003. Portanus Ball: descrições de dez espécies novas (Hemiptera, Cicadellidae, Xestocephalinae). Revista Brasileira de Entomologia 47: 547-558.

Carvalho, A. N. \& R. R. Cavichioli. 2004a. Uma nova espécie de Portanus Ball (Hemiptera, Cicadellidae) do Brasil. Revista Brasileira de Entomologia 48: 447-448.

Carvalho, A. N. \& R. R. Cavichioli. 2004b. Portanus Ball: descrição de uma espécie nova (Hemiptera, Cicadellidae, Xestocephalinae). Revista Brasileira de Entomologia 48: 339-341.

Carvalho, A. N. \& R. R. Cavichioli. 2005. Portanus aliceae sp. nov. do Brasil (Hemiptera: Cicadellidae, Xestocephalinae). Neotropical Entomology 34: 251-254.

Carvalho, A. N. \& R. R. Cavichioli. 2006. Taxonomia e Análise Cladística do Gênero Portanus Ball, 1932 (Hemiptera,
Cicadellidae, Xestocephalinae). Tese de doutorado Universidade Federal do Paraná, Curitiba-PR. 152 pp.

Davis, R. B. 1975. Classification of selected higher categories of auchenorrhynchous Homoptera (Cicadellidae and Aetalionidae). Technical Bulletin of the United States Department of Agriculture 1494: 1-52.

DeLong, D. M. 1976. New species of Portanus (Homoptera, Cicadellidae) from Bolivia. Brenesia 9: 37-49.

DeLong, D. M. 1980. New South American Xestocephalinae leafhoppers (Homoptera: Cicadellidae). Entomological News 91: 79-84.

DeLong, D. M. \& R. E. Linnavuori. 1978. Studies on Neotropical leafhoppers (Homoptera: Cicadellidae). Entomological Scandinavia 9: 111-123.

Hamilton, K. G. A. 1981. Morphology and evolution of the rhynchotan head (Insecta: Hemiptera, Homoptera). Canadian Entomologist 113: $953-794$.

Hill, B. G. 1970. Comparative morphological study of selected higher categories of leafhoppers (Homoptera: Cicadellidae). University Microfilms, Ann Arbor, xi +187 p.

Kramer, J. P. 1961. New Venezuelan leafhoppers of the subfamilies Xestocephalinae and Neocoelidinae (Homoptera: Cicadellidae). Proceedings of the Biological Society of Washington 74: 235-239.

Kramer, J. P. 1964. A Key for Portanus with new records and descriptions of new species (Homoptera: Cicadellidae: Xestocephalinae). Proceedings of the Entomological Society of Washington 66: $5-11$.

Linnavuori, R. E. 1959. Revision of the Neotropical Deltocephalinae and some related subfamilies (Homoptera). Annual Zoology Society 'Vanamo' 20: 45-51.

Oman, P. W. 1949. The Neartic leafhoppers (Homoptera: Cicadellidae). A generic classification and check list. Memoirs of the Entomological Society of Washington 3: 1-253.

Osborn, H. 1923. Neotropical Homoptera of the Carnegie Museum II. Annual Carnegie Museum 15: 27-79.

Young, D. A. 1968. Taxonomic study of the Cicadellinae (Homoptera: Cicadellidae) Part 1. Proconiini. Bulletin of the United States National Museum 261: 1-287.

Young, D. A. 1977. Taxonomic study of the Cicadellinae (Homoptera: Cicadellidae) Part 2. New world Cicadellini and the Genus Cicadella. Technical Bulletin of the North Carolina Agricultural Experiment Station 239: 1-1135. 